\title{
SOBRE LAS FORMAS DE MINIMO VOLUMEN DE LAS CELOSÍAS DE SECCIÓN CONSTANTE
}

(ON MINIMUM VOLUME SHAPES OF TRUSS-BEAMS WITH CONSTANT CROSS SECTION)

Valentín Quintas Ripoll, Dept. Estructuras de Edificación

E.T.S. Arquitectura. Universidad Politécnica de Madrid ESPAÑA

$422-24$

Fecha de recepción: 1-X-91

\section{RESUMEN}

Se estudian en este artículo las formas de mínimo volumen en celosías de sección constante, sin tener en cuenta el efecto del pandeo. El estudio se limita al caso de vigas apoyadas con cargas iguales, pero puede generalizarse fácilmente a otros tipos de sustentación y de carga.

\section{SUMMARY}

Minimum volume forms of truss-beams with constant cross section, are studied in this paper. The study is restrained only to simply supported beams, neglecting the effect of buckling, but it can be easily generalised to other types, such as continuos beams or cantilevers.

\section{INTRODUCCIÓN}

Una estructura utilizada muy a menudo en edificación, es la celosía triangulada de canto constante formada por dos cordones paralelos encargados de resistir el momento, unidos por un triangulado que resiste al cortante.

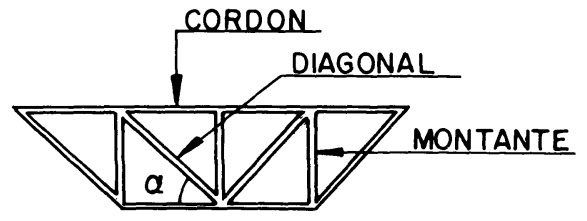

Habitualmente las barras paralelas - "los cordones" - son de una sección constante y dimensionadas para resistir el momento máximo; mientras que las barras del triangulado - "los montantes" y "las diagonales"-, están dimensionadas para resistir el cortante máximo.

Estas estructuras tienen una inclinación de diagonales y, por tanto, una relación entre la luz y el canto ópti$\mathrm{ma}$, para la cual el consumo de material es mínimo. Si se desprecian el efecto de pandeo y los saltos de catálogo de los perfiles, este mínimo no depende de la luz ni de la carga, sino exclusivamente del número de recuadros que forman la celosía. Aunque se han conseguido hasta ahora resultados parciales, como los de Aroca (1) suponiendo canto fijo, o los de Farkas (2) para algún caso particular, no se han obtenido estas formas de modo generalizado, a pesar de la sencillez de su desarrollo teórico como vamos a ver a continuación. 


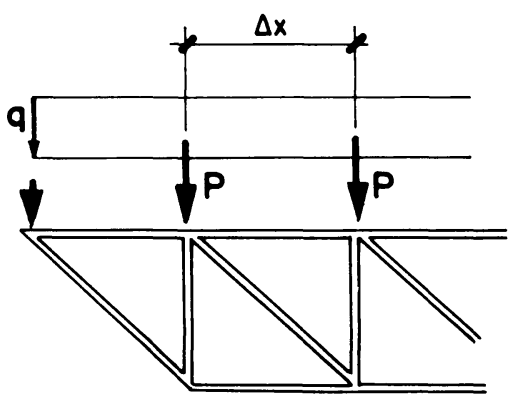

Supondremos en adelante que la celosía está simplemente apoyada y sometida a una serie de cargas iguales $P$, producidas por unas correas apoyadas en los nudos superiores que, a su vez, resisten una carga uniforme q, de forma que:

$$
P=q \cdot \Delta x
$$

si $\Delta \mathrm{x}$ es la separación de las correas.

A partir de los resultados de este caso es fácil generalizar a los de voladizos, vigas continuas, pórticos, etc. que, para evitar una extensión excesiva, no se estudian en este artículo.

\section{CELOSÍA DE DIAGONALES}
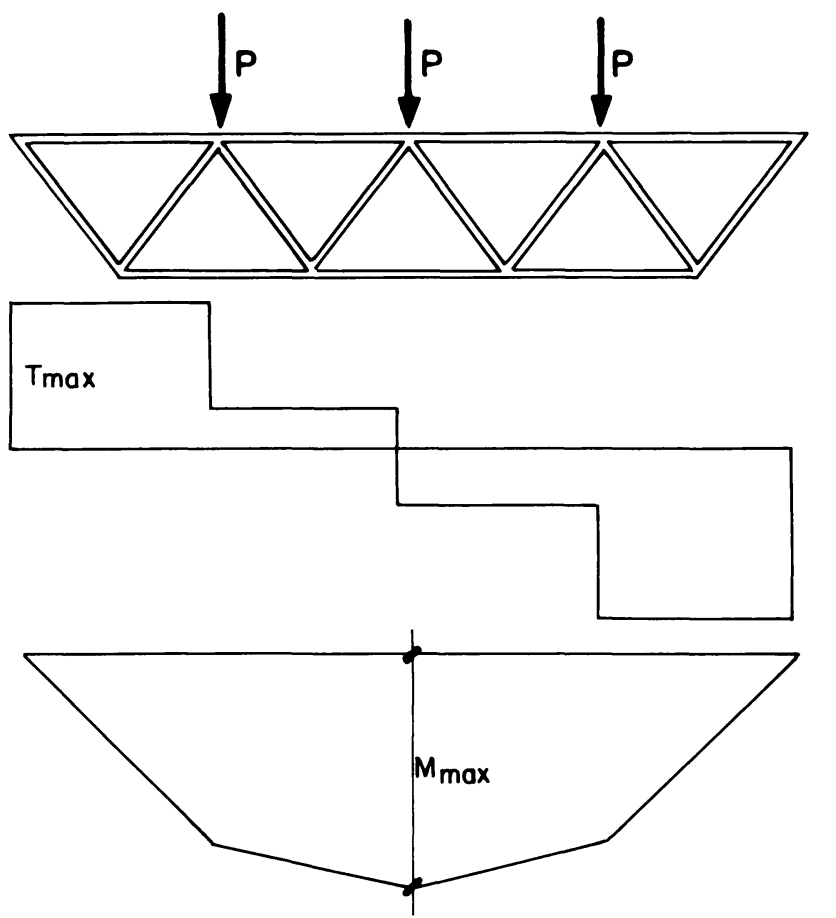

Empecemos por el caso más sencillo: la celosía cuyo triangulado está formado exclusivamente por una familia de diagonales inclinadas un ángulo $\alpha$ respecto a la horizontal.

Si es de sección constante, los cordones se dimensionarán con el momento máximo (3), y las diagonales con el cortante máximo. Supongamos que el número de cargas $\mathrm{P}$ que actúan en los nudos superiores es $\mathrm{n}_{\mathrm{C}}$. El volumen $\mathrm{V}$ de las barras de la celosía será:

$$
V=\left(2 n_{C}+1\right) V_{C}+2\left(n_{C}+1\right) V_{D}
$$

si llamamos $V_{C}$ al volumen de cada una de las barras que forman el cordón y $V_{D}$ al de cada una de las diagonales.

El volumen de una barra es el producto de su área por su longitud. En el caso de una de las del cordón:

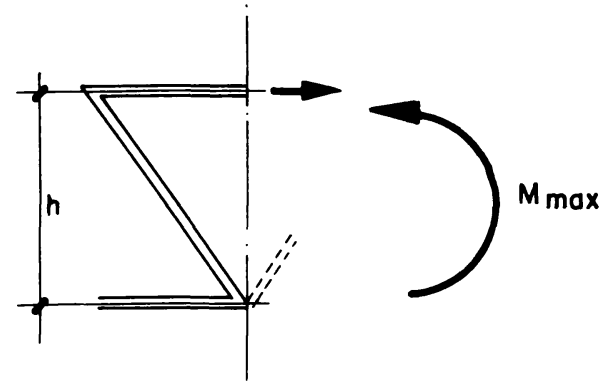

$$
\begin{gathered}
V_{c}=A_{c} \cdot I_{c} \\
I_{c}=\Delta x \\
A_{c}=\frac{N_{c}}{\sigma}=\frac{M_{\max }}{h \cdot \sigma}=\frac{M_{\max }}{\frac{\Delta x}{2} \cdot \tan \alpha \cdot \sigma}
\end{gathered}
$$

si llamamos $\mathrm{N}_{C}$ al normal máximo que soporta el cordón y, que se obtiene fácilmente tomando momentos en una sección respecto a un nudo; $h$ el canto de la celosía, y $\sigma$ la tensión a que trabaja la barra, que supondremos que es la misma a tracción y a compresión. 
En la diagonal:

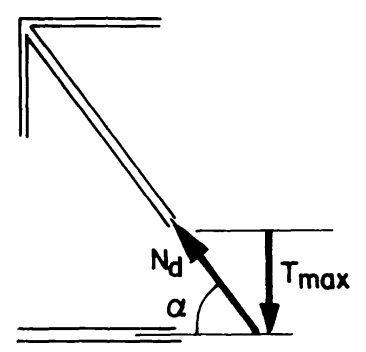

$$
\begin{aligned}
& V_{d}=A_{d} \cdot I_{d} \\
& I_{d}=\frac{\Delta x}{2} \cdot \frac{1}{\cos \alpha} \\
& A_{d}=\frac{N_{d}}{\sigma}=\frac{T_{\max }}{\sigma \cdot \sin \alpha}
\end{aligned}
$$

lo que se obtiene planteando el equilibrio de fuerzas verticales en una sección.

Sustituyendo sucesivamente, sacando factor común y sabiendo que:

$$
\frac{1}{\sin \alpha \cdot \cos \alpha}=\frac{1}{\tan \alpha}+\tan \alpha
$$

queda:

$$
V=\frac{\Delta x}{\sigma} \cdot\left(n_{c}+1\right) \cdot T_{\max }\left[\left(2 \cdot \frac{2 n_{c}+1}{n_{c}+1} \cdot \frac{M_{\max }}{T_{\max } \cdot \Delta x}+1\right) \cdot \frac{1}{\tan \alpha}+\tan \alpha\right]
$$

El volumen en función de un único parámetro $\operatorname{tg} \alpha$ que define la inclinación de las diagonales y, por tanto, el canto. Si derivamos $V$ respecto a $\operatorname{tg} \alpha$ e igualamos a cero obtenemos el valor $\operatorname{tg} \alpha_{0}$ de la tangente que hace mínimo el volumen:

$$
\frac{\delta V}{\delta(\tan \alpha)}=0=-\left(2 \cdot \frac{2 n_{c}+1}{n_{c}+1} \cdot \frac{M_{\max }}{T_{\max } \cdot \Delta x}+1\right) \cdot \frac{1}{\tan ^{2} \cdot \alpha}+1
$$

y queda:

$$
\tan \alpha_{0}=\sqrt{2 \cdot \frac{2 n_{c}+1}{n_{c}+1} \cdot \frac{M_{\max }}{T_{\max } \cdot \Delta x}+1}
$$


En el caso particular en el que todas las cargas sean iguales y separadas una misma distancia $\Delta x$, el cortante $T_{\max }$ es sencillamente la suma de las cargas dividida por dos:
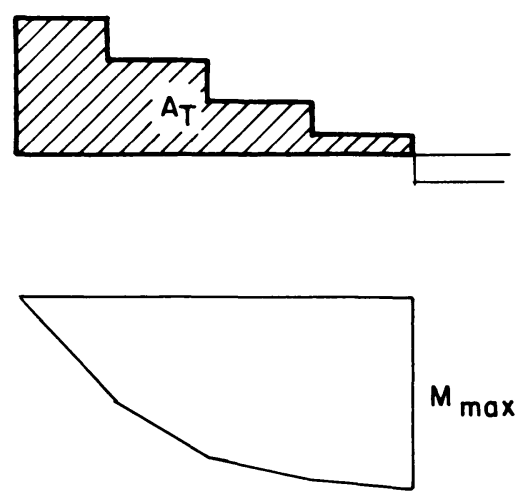

$$
T_{\max }=\frac{n_{c} \cdot P}{2}
$$

El momento máximo puede obtenerse como el área del diagrama de cortantes comprendido entre el apoyo y el centro de la celosía:

$$
M_{\max }=A_{T}=\sum_{1,1 / 2}^{n_{c} / 2} \cdot T_{n} \cdot \Delta x=\frac{n_{c}}{2} \cdot p \cdot \Delta x+\left(\frac{n_{c}}{2}-1\right) p \cdot \Delta x+\left(\frac{n_{c}}{2}-2\right) \cdot p \cdot \Delta x+\ldots \ldots+p \cdot \Delta x
$$

Esta suma es la de una progresión aritmética de $\frac{n_{C}}{2}$ términos si $n_{C}$ es par, y de $\frac{n_{C}+1}{2}$ términos si $n_{C}$ es impar, con lo que podemos obtener directamente su valor:

$$
\begin{array}{ll}
M_{\max }=n_{C} \frac{n_{C}+2}{8} p \Delta x & \text { si } n_{C} \text { es par } \\
M_{\max }=\frac{\left(n_{C}+1\right)^{2}}{8} p \Delta x & \text { si } n_{C} \text { es impar }
\end{array}
$$

Si ahora sustituimos en la ecuación de la tangente, queda:

$$
\begin{aligned}
& \tan \alpha_{0}=\sqrt{\frac{\left(2 n_{c}+1\right) \cdot\left(n_{c}+2\right)}{2\left(n_{c}+1\right)}+1} \quad \text { si } n_{c} \text { es par } \\
& \tan \alpha_{0}=\sqrt{\frac{\left(2 n_{c}+1\right)\left(n_{c}+1\right)}{2 n_{c}}+1} \quad \text { si } n_{c} \text { es impar }
\end{aligned}
$$

Y si sustituimos en la fórmula del volumen queda:

$$
V_{\min }=\frac{q l^{2}}{\sigma} \cdot \frac{n_{c}}{n_{c}+1} \cdot \tan \alpha
$$


teniendo en cuenta que $\Delta x=\frac{L}{n_{C}+1}$, si $L$ es la luz de la celosía, y vemos que, al igual que ocurre en todas las estructuras que resisten una carga uniforme $q(4)$, el volumen mínimo de material es proporcional a $q$, al cuadrado de la luz, a la inversa de la tensión de trabajo y a un "factor de forma" $F$ adimensional, que sólo depende del número de recuadros, o de cargas $n_{c}$ :

$$
V_{\min }=\frac{q l^{2}}{\sigma} \cdot F
$$

y:

$$
F=\frac{n_{c}}{n_{c}+1} \cdot \tan \alpha_{0}
$$

En nuestro caso $\mathrm{F}$ es el único término que nos interesa, ya que es el único que depende de la forma. Las celosías de mínimo material, para cada caso, son entonces las siguientes:

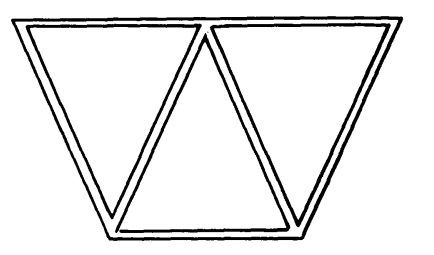

$$
\mathrm{n}_{\mathrm{c}}=1 \quad \operatorname{tg} \alpha_{0}=2 \quad \mathrm{v}=\frac{\mathrm{ql}^{2}}{\sigma} \quad \alpha_{0}=63,43^{\circ} \quad \lambda=2
$$

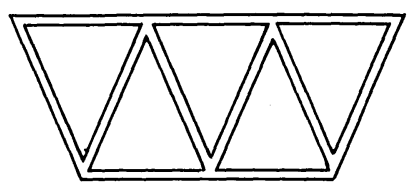

$$
n_{c}=2 \quad \operatorname{tg} \alpha_{0}=\sqrt{\frac{13}{3}}
$$

$$
v=1,39 \frac{q l^{2}}{\sigma}
$$

$\alpha_{0}=64,34^{\circ}$

$\lambda=2,88$

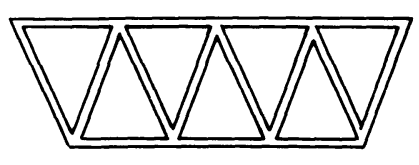

$$
\mathrm{n}_{\mathrm{c}}=3 \operatorname{tg} \alpha_{0}=\sqrt{\frac{17}{3}}
$$

$$
v=1,379 \frac{q l^{2}}{\sigma}
$$

$\alpha_{0}=67,21^{\circ}$

$\lambda=3,36$

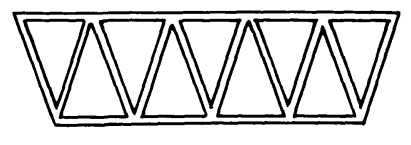

$$
\mathrm{n}_{\mathrm{c}}=4 \quad \operatorname{tg} \alpha_{0}=\sqrt{\frac{32}{5}}
$$

$$
v=2,02 \frac{q l^{2}}{\sigma}
$$

$\alpha_{0}=68,43^{\circ}$

$\lambda=3,95$

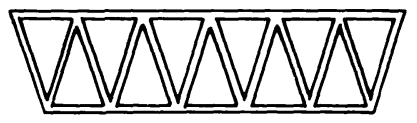

$$
\mathrm{n}_{\mathrm{c}}=5 \quad \operatorname{tg} \alpha_{0}=\sqrt{\frac{38}{5}}
$$

$$
v=2,3 \frac{\mathrm{ql}^{2}}{\sigma}
$$

$\alpha_{0}=70^{\circ}$

$$
\lambda=4,35
$$


Como puede observarse, los ángulos óptimos son siempre superiores a $60^{\circ}$, y crecientes con $\mathrm{n}_{\mathrm{C}}$. En el límite, cuando $\mathrm{n}_{\mathrm{C}} \rightarrow \infty, d \rightarrow 90^{\circ}$. El volumen crece también con $\mathrm{n}_{\mathrm{C}}$, aunque no linealmente, y lo mismo ocurre con la "esbeltez" $\lambda$, es decir, con la relación entre la luz $L$ y el canto $h$, en efecto:

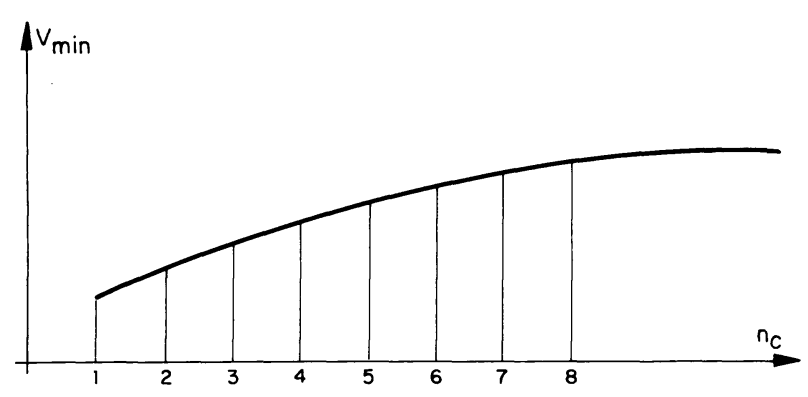

$$
\lambda=L_{h}=\frac{\left(n_{c}+1\right) \cdot \Delta x}{\frac{\Delta x}{2} \cdot \tan \alpha_{0}}=\frac{2\left(n_{c}+1\right)}{\tan \alpha_{0}}
$$

Para $n_{C}$ altos la forma óptima empezaría a plantear problemas de flecha, al crecer el numerador linealmente con $\mathrm{n}_{\mathrm{C}}$ y no hacerlo tg $\alpha_{0}$.

Veamos qué resultados podemos deducir para otros triangulados.

\section{CELOSÍA DE PIRÁMIDE CUADRADA}

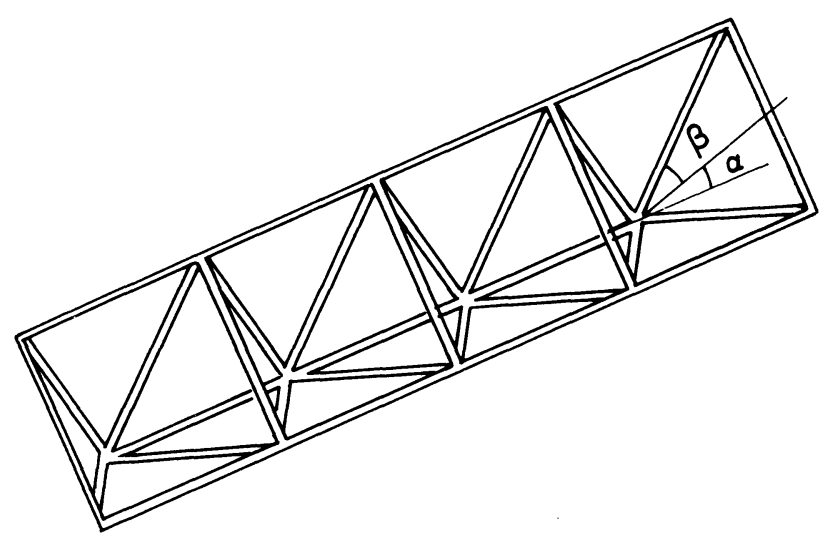

Es una celosía espacial que se puede generar a partir de dos celosías de diagonales inclinadas un ángulo $\beta$ respecto al plano vertical. Si se unen sus nudos superiores por una serie de barras horizontales, sus barras limitan una serie de pirámides invertidas. Su uso es cada vez más frecuente porque, al ser una estructura espacial, se eliminan los problemas de pandeo general de celosías que trabajan en un plano. 
Su volumen se obtiene fácilmente a partir del de las diagonales, si tenemos en cuenta que el número de barras que forman las diagonales y el cordón superior es directamente el doble, y se desprecia el volumen de las barras horizontales que unen las dos celosías, ya que no trabajan.

Si $V_{C} s$ es el volumen de un cordón superior, $y V_{c}{ }^{\prime}$, el de un cordón inferior queda:

$$
V=2\left(n_{C}+1\right) V_{C}^{S}+n_{C} \cdot V_{C}^{I}+4\left(n_{C}+1\right) V_{D}
$$

Si ahora sustituimos:

$$
\begin{gathered}
V_{C}^{S}=\frac{M_{\max }}{\Delta x \cdot \tan \alpha \sigma} \cdot \Delta x \\
V_{C}^{I}=\frac{2 M_{\max }}{\Delta x \cdot \tan \alpha \sigma} \cdot \Delta x \\
V_{D}=\frac{N_{D}}{\sigma} \cdot I_{D}=\frac{T_{\max }}{2 \sin \alpha \cdot \cos \beta \cdot \sigma} \cdot \frac{\Delta x}{2 \cos \alpha \cdot \cos \beta}
\end{gathered}
$$$$
\text { (1) }
$$

Ya que el normal en una diagonal puede obtenerse descomponiendo entre dos diagonales la componente del cortante, $\frac{T_{\max }}{\operatorname{sen} \alpha}$ en su plano, queda después de simplificar:

$$
V=\left(n_{c}+1\right) \cdot \frac{T_{\max } \cdot \Delta x}{\sigma} \cdot\left[\left(2 \cdot \frac{2 n_{c}+1}{n_{c}+1} \cdot \frac{M_{\max }}{T_{\max } \cdot \Delta x}+\frac{1}{\cos ^{2} \beta}\right) \cdot \frac{1}{\tan \alpha}+\frac{\tan \alpha}{\cos ^{2} \beta}\right]
$$

Vemos que la expresión sería la misma que la de la viga de diagonales, si hiciésemos $\cos \beta=1$. El término $\cos \beta$, hace el volumen mayor, con lo que desde el punto de vista teórico no resulta ninguna ventaja en hacer la celosía en pirámide.

En el caso particular en que la pirámide sea cuadrada, se verifica:

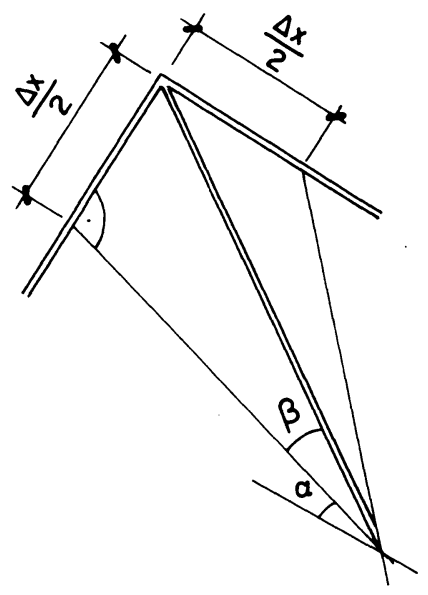

(c) Consejo Superior de Investigaciones Científicas Licencia Creative Commons 3.0 España (by-nc)

$$
\begin{aligned}
& \frac{\Delta x}{2 \tan \beta}=\frac{\Delta x}{2 \cos \alpha} \quad y \quad \cos \alpha=\tan \beta \\
& \frac{1}{\cos ^{2} \beta}=1+\tan ^{2} \beta=1+\cos ^{2} \alpha \\
& \frac{\tan \alpha}{\cos ^{2} \beta}=\tan \alpha+\sin \alpha \cdot \cos \alpha \\
& \frac{1}{\tan \alpha} \cdot \frac{1}{\cos ^{2} \beta}=\frac{2}{\tan \alpha}-\sin \alpha \cdot \cos \alpha \\
& \frac{\tan \alpha}{\cos ^{2} \beta}+\frac{1}{\tan \alpha} \cdot \frac{1}{\cos ^{2} \beta}=\tan \alpha+\frac{2}{\tan \alpha}
\end{aligned}
$$


Con lo que queda:

$$
V=\left(n_{c}+1\right) \cdot \frac{T_{\max } \cdot \Delta x}{\sigma}\left[\left(2 \cdot \frac{2 n_{c}+1}{n_{c}+1} \cdot \frac{M_{\max }}{T_{\max } \cdot \delta x}+2\right) \cdot \frac{1}{\tan \alpha}+\tan \alpha\right]
$$

El volumen en función de $\operatorname{tg} \alpha$. Si derivamos con respecto a $\operatorname{tg} \alpha$, e igualamos a cero, obtenemos el parámetro óptimo:

$$
\tan \alpha_{0}=\sqrt{\frac{\left(2 n_{c}+1\right)\left(n_{c}+2\right)}{2\left(n_{c}+1\right)}+2}
$$

que es siempre mayor que el de la celosía de diagonales. El volumen mínimo es:

$$
V_{\min }=2\left(n_{c}+1\right) \cdot \frac{T_{\max } \cdot \Delta x}{\sigma} \cdot \tan \alpha_{0}
$$

En el caso particular en que todas las cargas sean iguales, queda siguiendo el razonamiento de la celosía de diagonales:

$$
\begin{gathered}
\tan \alpha_{0}=\sqrt{\frac{\left(2 n_{c}+1\right)\left(n_{c}+2\right)}{2\left(n_{c}+1\right)}+2} \quad \text { si } n_{c} \text { es par } \\
\tan \alpha_{0}=\sqrt{\frac{\left(2 n_{c}+1\right)\left(n_{c}+1\right)}{2 n_{c}}+2} \quad \text { si } n_{c} \text { es impar } \\
V_{\min }=\frac{n_{c}}{n_{c}+1} \cdot \tan \alpha_{0} \cdot \frac{q l^{2}}{\sigma}
\end{gathered}
$$

el "factor de forma" tiene la misma expresión que en la celosía de diagonales, y lo mismo ocurre con la esbeltez.

Las formas óptimas para esta celosía son entonces: 


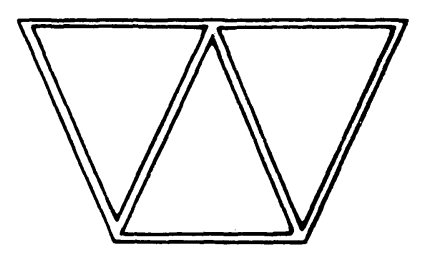

$\mathrm{n}_{\mathrm{c}}=1 \quad \operatorname{tg} \alpha_{0}=\sqrt{5} \quad \alpha_{0}=65,9^{\circ} \quad \mathrm{v}=1,12 \frac{\left.\mathrm{q}\right|^{2}}{\sigma} \quad \lambda=\frac{4}{\sqrt{ } 5}=1,79$

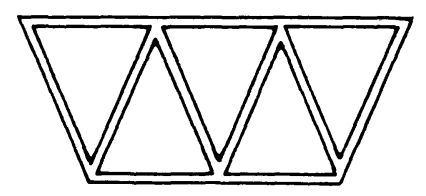

$\mathrm{n}_{\mathrm{c}}=2 \quad \operatorname{tg} \alpha_{0}=\frac{4}{\sqrt{3}} \quad \alpha_{0}=66,5^{\circ} \quad \mathrm{v}=1,53 \frac{\left.\mathrm{q}\right|^{2}}{\sigma} \quad \lambda=2,60$

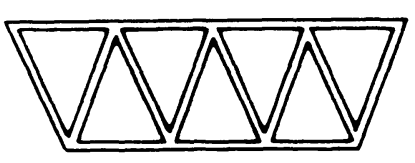

$\mathrm{n}_{\mathrm{c}}=3 \quad \operatorname{tg} \alpha_{0}=\frac{2 \sqrt{5}}{\sqrt{3}}$

$\alpha_{0}=68,82^{\circ}$

$v=1,94 \frac{\mathrm{q}^{2}}{\sigma} \quad \lambda=3,10$

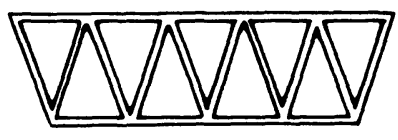

$\mathrm{n}_{\mathrm{c}}=4 \quad \operatorname{tg} \alpha_{0}=\sqrt{\frac{37}{5}}$

$\alpha_{0}=69,8^{\circ}$

$v=2,17 \frac{\left.q\right|^{2}}{\sigma} \quad \lambda=3,67$

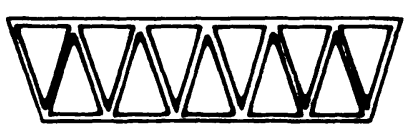

$\mathrm{n}_{\mathrm{c}}=5 \quad \operatorname{tg} \alpha_{0}=\sqrt{\frac{43}{5}}$

$\alpha_{0}=71,17^{\circ}$

$v=2,44 \frac{\mathrm{ql}^{2}}{\sigma} \quad \lambda=4,09$

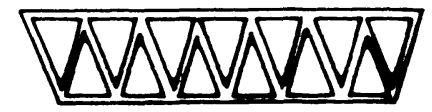

$\mathrm{n}_{\mathrm{c}}=6 \quad \operatorname{tg} \alpha_{0}=\sqrt{\frac{66}{7}}$

$\alpha_{0}=71,96^{\circ}$

$v=2,63 \frac{q 1^{2}}{\sigma} \quad \lambda=4,56$

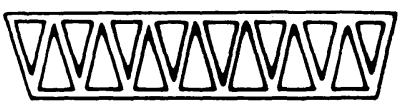

$\mathrm{n}_{\mathrm{c}}=7 \quad \operatorname{tg} \alpha_{0}=\sqrt{\frac{74}{7}}$

$\alpha_{0}=72,9^{\circ}$

$v=2,84 \frac{q l^{2}}{\sigma} \quad \lambda=4,92$

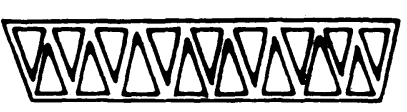

$$
\mathrm{n}_{\mathrm{c}}=8 \quad \operatorname{tg} \alpha_{0}=\sqrt{\frac{103}{9}}
$$

$\alpha_{0}=73,5^{\circ}$

$v=3 \frac{\left.q\right|^{2}}{\sigma} \quad \lambda=5,32$ 
Si comparamos estos valores con los de la celosía de diagonales, vemos que el volumen es mayor, aunque sólo ligeramente (un $5 \%$ como media), por lo que las ventajas que discutíamos podrían hacer más aconsejable este tipo. Los ángulos y esbelteces son muy semejantes en los dos casos, sobre todo para valores de $\mathrm{n}_{\mathrm{C}}$ altos.

\section{CELOSÍA DE DIAGONALES Y MONTANTES}

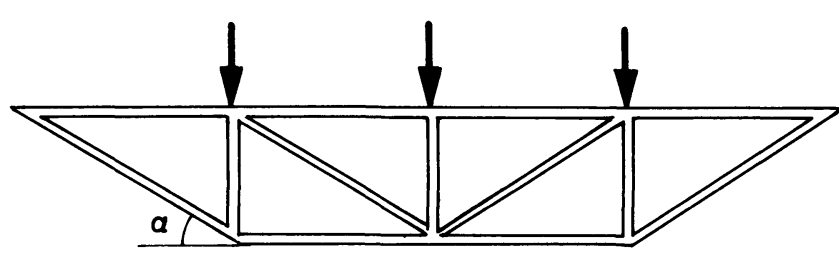

Es la muy conocida solución que se utiliza cuando se quiere que todas las diagonales trabajen a tracción, si el pandeo de las diagonales empieza a ser importante. A cambio deben colocarse unos montantes verticales, que trabajan con un esfuerzo de compresión igual al cortante que soporta la diagonal que concurre en su nudo inferior.

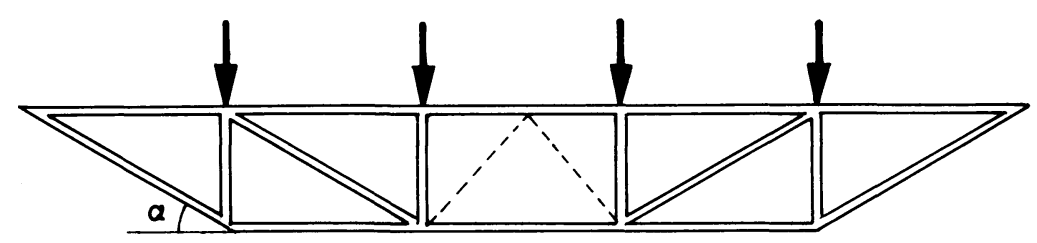

Si llamamos $V_{m}, N_{m}$ y $I_{m}$ al volumen, normal y longitud del montante, el volumen de la celosía es:

$$
\begin{gathered}
V=2 n_{c} \cdot V_{c}+\left(n_{c}+1\right) \cdot V_{d}+n_{c} \cdot V_{m} \quad \text { si } n_{c} \text { es impar } \\
V=2 n_{c} \cdot V_{c}+n_{c} \cdot V_{d}+n_{c} \cdot V_{m} \quad \text { si } n_{c} \text { es par, }
\end{gathered}
$$

suponiendo que no se colocan diagonales en el recuadro central, al ser en esa zona el cortante nulo. En la realidad habría que colocar un triangulado que resistiese el cortante debido a una sobrecarga no simétrica, pero supondremos que el área de ese triangulado es despreciable.

El volumen del cordón será igual que en el primer caso:

$$
V_{c}=\frac{M_{\max }}{\sigma \cdot \Delta x \cdot \tan \alpha} \cdot \Delta x
$$

El volumen de una diagonal:

$$
V_{D}=\frac{T_{\max } \cdot \Delta x}{\sigma}\left(\frac{1}{\tan \alpha}+\tan \alpha\right)
$$

Finalmente el volumen de un montante:

$$
V_{m}=A_{m} \cdot I_{m}=\frac{N_{m}}{\sigma} \cdot I_{m}=\frac{T_{\max }}{\sigma} \cdot \Delta x \cdot \tan \alpha
$$


a excepción del caso de $\mathrm{n}_{\mathrm{C}}=1$, en el que el único montante trabaja con $2 T_{\max }$, en lugar de $T_{\max }$. Sustituyendo en las ecuaciones del volumen, resulta:

$$
\begin{aligned}
& V=\frac{2 n_{c} \cdot T_{\max } \cdot \Delta x}{\sigma}\left[\left(\frac{M_{\max }}{T_{\max } \cdot \Delta x}+\frac{1}{2}\right) \cdot \frac{1}{\tan \alpha}+\tan \alpha\right] \quad \text { si } n_{c} \text { es par } \\
& V=\frac{\left(2 n_{c}+1\right) \cdot T_{\max } \cdot \Delta x}{\sigma}\left[\left(\frac{2 n_{c}}{2 n_{c}+1} \cdot \frac{M_{\max }}{T_{\max } \cdot \Delta x}+\frac{n_{c}+1}{2 n_{c}+1}\right) \cdot \frac{1}{\tan \alpha}+\tan \alpha\right]
\end{aligned}
$$

si $n_{C}$ es impar, a excepción del caso en que $n_{C}=1$.

Derivando con respecto al parámetro $\operatorname{tg} \alpha$ e igualando a cero, obtenemos los valores de los parámetros que hacen el volumen mínimo:

$$
\begin{gathered}
\tan \alpha_{0}=\sqrt{\frac{M_{\max }}{T_{\max } \cdot \Delta x}+\frac{1}{2}} \quad \text { si } n_{C} \text { es par } \\
V_{\min }=\frac{4 n_{c} \cdot T_{\max } \cdot \Delta x}{\sigma} \cdot \tan \alpha_{0} \quad \\
\tan \alpha_{0}=\sqrt{\frac{2 n_{c}}{2 n_{c}+1} \cdot \frac{M_{\max }}{T_{\max } \cdot \Delta x}+\frac{n_{c}+1}{2 n_{c}+1}} \text { si } n_{C} \text { es impar } \\
V_{\min }=2\left(2 n_{c}+1\right) \cdot \frac{T_{\max } \cdot \Delta x}{\sigma} \cdot \tan \alpha_{0}
\end{gathered}
$$

En el caso en que todas las cargas sean iguales, resulta:

$$
\begin{aligned}
& \tan \alpha_{0}=\frac{\sqrt{n_{c}+4}}{2} \\
& V_{\min }=\frac{2 n_{c}^{2}}{\left(n_{c}+1\right)^{2}} \cdot \tan \alpha_{0} \frac{q l^{2}}{\sigma} \\
& \tan \alpha_{0}=\sqrt{\frac{\left(n_{c}+1\right)\left(n_{c}+3\right)}{2\left(2 n_{c}+1\right)}} \quad \text { si } n_{c} \text { es par } \\
& V_{\min }=\frac{\left(2 n_{c}+1\right) \cdot n_{c}}{\left(n_{c}+1\right)^{2}} \cdot \tan \alpha_{0} \cdot \frac{q l^{2}}{\sigma}
\end{aligned}
$$


La esbeltez $\lambda$ es:

$$
\lambda=\frac{L}{h}=\frac{n_{c}+1}{\tan \alpha_{0}}
$$

en ambos casos

el caso en que $n_{c}=1$ se obtiene fácilmente duplicando el volumen del montante. Las formas de mínimo volumen son entonces las siguientes:

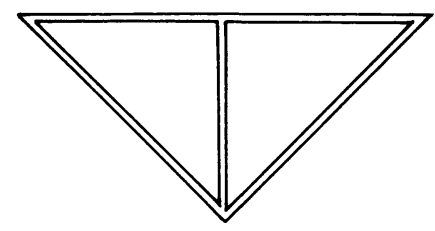

$$
\mathrm{n}_{\mathrm{c}}=1 \quad \operatorname{tg}_{0}=1 \quad \alpha_{0}=45^{\circ} \quad \mathrm{v}=\frac{\mathrm{q}^{2}}{\sigma} \quad \lambda=2
$$

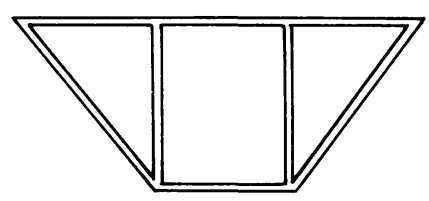

$\mathrm{n}_{\mathrm{c}}=2 \quad \operatorname{tg} \alpha_{0}=\frac{\sqrt{3}}{\sqrt{2}} \quad \alpha_{0}=50,76^{\circ} \quad \mathrm{v}=1,088 \frac{\mathrm{ql}^{2}}{\sigma} \quad \lambda=2,45$

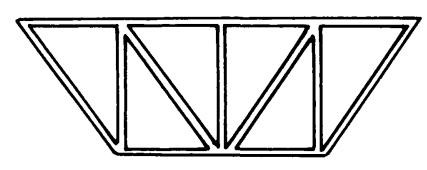

$$
\mathrm{n}_{\mathrm{c}}=3 \quad \operatorname{tg} \alpha_{0}=\frac{2 \sqrt{3}}{\sqrt{7}} \quad \alpha_{0}=52,63^{\circ} \quad \mathrm{v}=1,71 \frac{\mathrm{ql}^{2}}{\sigma} \quad \lambda=3,05
$$

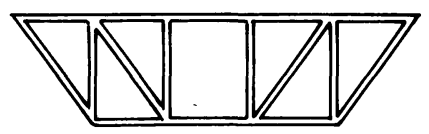

$\mathrm{n}_{\mathrm{c}}=4 \quad \operatorname{tg} \alpha_{0}=\sqrt{2}$

$\alpha_{0}=54,73^{\circ}$

$v=1,81 \frac{q l^{2}}{\sigma}$

$\lambda=3,53$

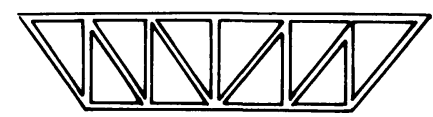

$\mathrm{n}_{\mathrm{c}}=5 \quad \operatorname{tg} \alpha_{0}=\sqrt{\frac{24}{11}} \quad \alpha_{0}=55,9^{\circ}$

$v=2,25 \frac{\mathrm{ql}^{2}}{\sigma} \quad \lambda=4,06$

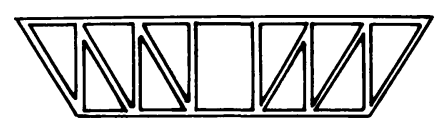

$\mathrm{n}_{\mathrm{c}}=6 \quad \operatorname{tg} \alpha_{0}=\sqrt{\frac{5}{2}}$

$\alpha_{0}=57,69^{\circ}$

$v=2,32 \frac{\mathrm{ql}^{2}}{\sigma}$

$\lambda=4,43$ 


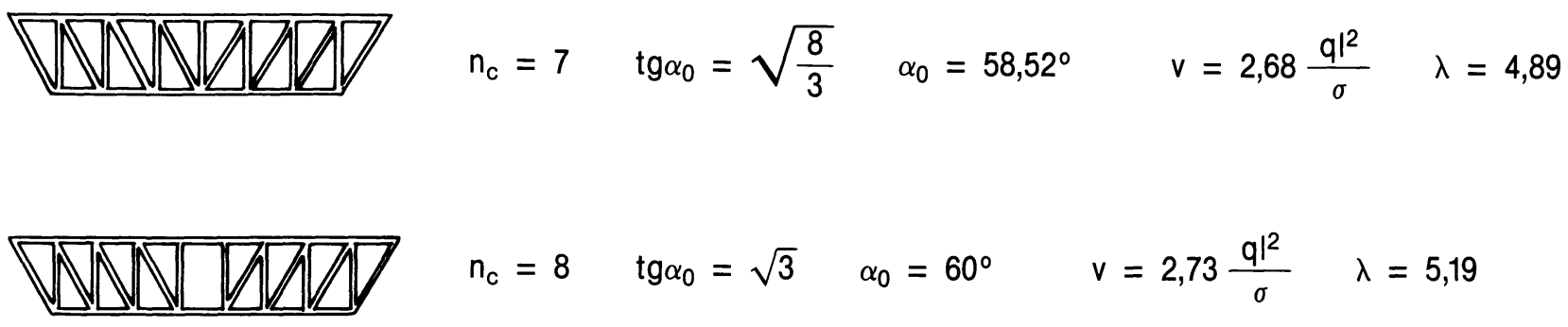

Los dos primeros casos $\left(n_{C}=1\right.$ y $\left.n_{C}=2\right)$ tienen un volumen extraordinariamente pequeño al ser formas antifuniculares de la carga, y coinciden con las ya obtenidas en otro trabajo (5). A partir de $n_{C}=3$ el volumen empieza a crecer rápidamente con $n_{C} y$, aunque siempre se mantiene menor que el de los casos anteriores, la diferencia es cada vez más pequeña. Cuando $n_{C}=8$, ésta es ya menor del $5 \%$.

Aparentemente una mayor complicación en el diseño del triangulado no aumenta el volumen de la estructura. Intentemos confirmarlo con el caso siguiente.

\section{CELOSÍA DE DOBLE DIAGONAL}

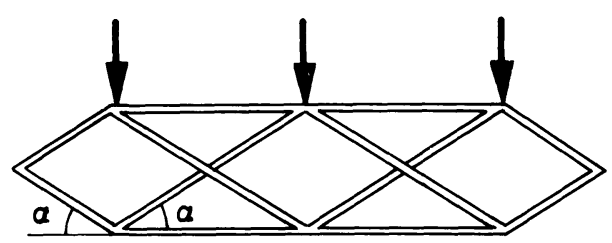<smiles>c1ccc(-c2ccco2)cc1</smiles>

En este diseño se utilizan dos familias de diagonales de igual inclinación. De esta forma se consigue que, en cada sección, el cortante esté soportado por dos diagonales, en lugar de por una.

Planteando el equilibrio en uno de los nudos centrales y en una sección se puede demostrar que se reparte en partes iguales entre cada familia.

Esta forma es interesante además porque es la base de las estructuras desplegables utilizadas por Piñero y sus seguidores: si retiramos los dos cordones tenemos una "tijera" desplegable.

El volumen es:

$$
V=2\left(n_{c}-1\right) \cdot V_{c}+2 n_{c} \cdot V_{D}
$$

Como:

$$
V_{c}=\frac{M_{\max }}{\sigma \cdot \Delta x \cdot \tan \alpha} \cdot \Delta x
$$

y:

$$
V_{D}=\frac{T_{\max }}{2 \sin \alpha \cdot \sigma} \cdot \frac{\Delta x}{\cos \alpha}=\frac{T_{\max }}{2 \sigma} \cdot \Delta x\left(\frac{1}{\tan \alpha}+\tan \alpha\right)
$$


si tenemos en cuenta que:

$$
\begin{aligned}
& N_{D}=\frac{T_{\max }}{2 \sin \alpha} \\
& I_{D}=\frac{\Delta x}{\cos \alpha}
\end{aligned}
$$

queda:

$$
V=n_{c} \cdot \frac{T_{\max } \cdot \Delta x}{\sigma} \cdot\left[\left(2 \cdot \frac{n_{c}-1}{n_{c}} \cdot \frac{M_{\max }}{T_{\max } \cdot \Delta x}+1\right) \cdot \frac{1}{\tan \alpha}+\tan \alpha\right]
$$

El valor de $\operatorname{tg} \alpha$ que hace mínimo $V$ es entonces:

$$
\tan \alpha_{0}=\sqrt{2 \cdot \frac{n_{c}-1}{n_{c}} \cdot \frac{M_{\max }}{T_{\max } \cdot \Delta x}+1}
$$

y el volumen mínimo:

$$
V_{\min }=2 n_{c} \cdot \frac{T_{\max } \cdot \Delta x}{\sigma} \cdot \tan \boldsymbol{\alpha}_{0}
$$

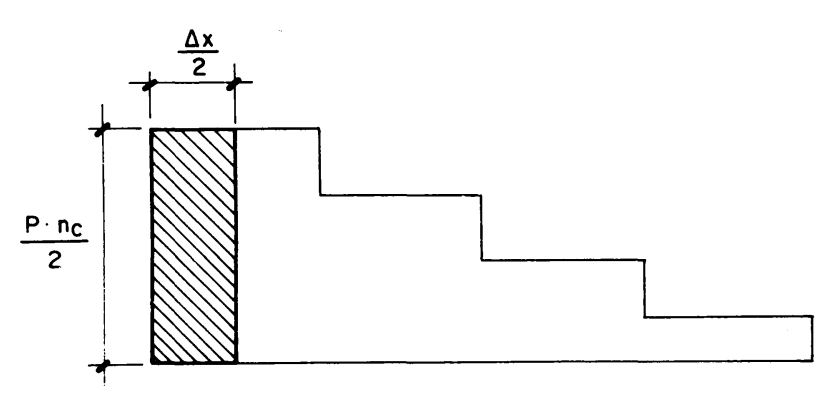

En el caso particular en que todas las cargas sean iguales, el cortante máximo continuaría siendo:

$$
T_{\max }=\frac{p \cdot n_{c}}{2}
$$

pero el momento sería menor al tener el primer recuadro una longitud de $\frac{\Delta \mathrm{x}}{2}$. Podemos deducir el valor del momento máximo restando al valor obtenido para los casos anteriores el área de cortantes en que se reduce $M_{\max }$

$$
\frac{p \cdot n_{c}}{2} \cdot \frac{\Delta x}{2}
$$

queda:

$$
\begin{array}{ll}
M_{\max }=p \cdot n_{c} \cdot \frac{n_{c}+2}{8} \cdot \Delta x-\frac{n_{c} \cdot p \cdot \Delta x}{4}=\frac{p \cdot n_{c}^{2}}{8} \cdot \Delta x & \text { si } n_{c} \text { es par, } y: \\
M_{\max }=\frac{p\left(n_{c}+1\right)^{2}}{8} \cdot \Delta x-\frac{n \cdot p \cdot \Delta x}{4}=p \cdot \frac{n_{c}^{2}+1}{8} \cdot \Delta x \quad \text { si } n_{c} \text { es impar. }
\end{array}
$$


Sustituyendo en las fórmulas de la tangente y del volumen, resulta:

$$
\begin{array}{cc}
\tan \alpha_{0}=\sqrt{\frac{n_{c}+1}{2}} & \text { si } n_{c} \text { es par } \\
\tan \alpha_{0}=\sqrt{\frac{\left(n_{c}-1\right)\left(n_{c}^{2}+1\right)}{2 n_{c}^{2}}+1} \quad \text { si } n_{c} \text { es impar }
\end{array}
$$

Y el volumen:

$$
V_{\min }=\frac{q l^{2}}{\sigma} \cdot \tan \alpha_{0}, \text { en ambos casos. }
$$

El factor de forma F, es directamente la tangente del ángulo óptimo.

Las formas de mínimo material son las siguientes, excluyendo la de $n_{C}=1$ que sería un mecanismo:

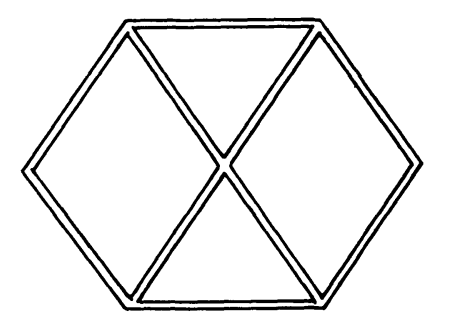

$$
\mathrm{n}_{\mathrm{c}}=2 \quad \operatorname{tg} \alpha_{0}=\sqrt{\frac{3}{2}} \quad \alpha_{0}=56,3^{\circ} \quad \mathrm{v}=1,22 \frac{\mathrm{q}^{2}}{\sigma} \quad \lambda=1,63
$$

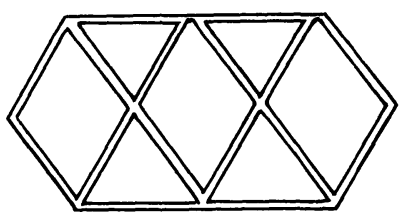

$$
\mathrm{n}_{\mathrm{c}}=3 \quad \operatorname{tg} \alpha_{0}=\sqrt{\frac{19}{3}} \quad \alpha_{0}=55,46^{\circ} \quad \mathrm{v}=1,45 \frac{\mathrm{ql}^{2}}{\sigma} \quad \lambda=2,06
$$

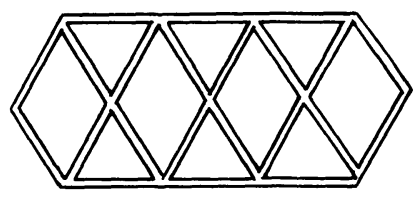

$$
\mathrm{n}_{\mathrm{c}}=4 \quad \operatorname{tg} \alpha_{0}=\sqrt{\frac{5}{2}} \quad \alpha_{0}=57,69^{\circ} \quad \mathrm{v}=1,58 \frac{\mathrm{q} \mathrm{l}^{2}}{\sigma} \quad \lambda=2,53
$$

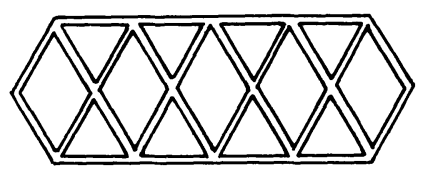

$$
\mathrm{n}_{\mathrm{c}}=5 \quad \operatorname{tg} \alpha_{0}=\sqrt{\frac{77}{5}} \quad \alpha_{0}=60,32^{\circ}
$$$$
v=1,75 \frac{\mathrm{q}^{2}}{\sigma} \quad \lambda=2,85
$$ 


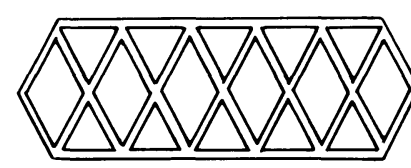

$$
\mathrm{n}_{\mathrm{c}}=6 \quad \operatorname{tg} \alpha_{0}=\sqrt{\frac{7}{2}} \quad \alpha_{0}=61,87^{\circ} \quad \mathrm{v}=1,87 \frac{\left.\mathrm{q}\right|^{2}}{\sigma} \quad \lambda=3,21
$$

$$
\mathrm{n}_{\mathrm{c}}=7 \quad \operatorname{tg} \alpha_{0}=\sqrt{\frac{199}{7}} \quad \alpha_{0}=63,60^{\circ} \quad \mathrm{v}=2,02 \frac{\mathrm{ql}^{2}}{\sigma} \quad \lambda=3,47
$$

$$
\mathrm{n}_{\mathrm{c}}=8 \quad \operatorname{tg} \alpha_{0}=\sqrt{\frac{9}{2}} \quad \alpha_{0}=64,76^{\circ} \quad \mathrm{v}=2,12 \frac{\left.\mathrm{q}\right|^{2}}{\sigma} \quad \lambda=3,77
$$

Excluyendo el caso de $n_{C}=2$, que en la celosía de diagonales y montantes coincidia con una forma antifunicular, este triangulado es el que menor volumen de material consume, a pesar de ser el de diseño menos sencillo.

El valor de la esbeltez es

$$
\lambda=\frac{n_{c}}{\tan \alpha_{0}}
$$

y es menor que en los casos anteriores aunque crece con $n_{C}$.

\section{CONCLUSIONES}

Del estudio de estos casos podemos deducir las siguientes conclusiones:

a) La obtención de las formas de mínimo volumen sin tener en cuenta el pandeo, en celosias de sección constante, es factible por optimización directa, aun en casos más complicados que los que hemos estudiado: vigas continuas, voladizos, etc.

b) El volumen de estructura es directamente proporcional a la carga uniforme que soporta q y al cuadrado de la luz, e inversamente proporcional a la tensión de trabajo $\sigma$. Igual conclusión se ha podido deducir para otras estructuras que soportan cargas uniformes en (4) y (5).

c) La influencia del diseño del triangulado en el volumen de ésta, puede reducirse a un "factor de forma" adimensional $\mathrm{F}$. Este factor crece rápidamente con el número de cargas $\mathrm{n}_{\mathrm{C}}$, de manera que a medida que $\mathrm{n}_{\mathrm{C}}$ aumenta, aumenta el volumen. Las celosias más económicas son pues las que menor número de recuadros tienen, si el efecto del pandeo no es importante.

d) Además, la esbeltez y la inclinación óptima de las diagonales crecen con $\mathrm{n}_{\mathrm{c}}$, hasta el punto que con un número de cargas elevado habría que dimensionar las formas óptimas a flecha y dejarian de ser volúmenes mínimos. 
e) La sencillez en el diseño de los triangulados, no implica una disminución del volumen total. En cualquier caso las diferencias de volumen entre un tipo y otro de triangulado no son lo suficientemente importantes como para que, en determinados casos, otros factores como la longitud de pandeo de las barras, 0 el coste de los nudos, no tengan más influencia en la elección del tipo.

Sin embargo, el aumento de sección por pandeo influye poco en los ángulos óptimos, como ya se ha demostrado en (5), al ser estructuras con cargas alineadas y, por tanto, con constante de Maxwell nula.

\section{REFERENCIAS Y NOTAS}

(1) AROCA, R.

"Diseño de Estructuras"

Apuntes inéditos del curso de Proyecto de Estructuras de la ETSAM.

(2) FARKAS, R.

"Optimal Structures".

(3) Aunque el cordón superior no se dimensionaría con el momento máximo, sino con un momento intermedio entre el máximo y el del nudo contiguo, hemos preferido darle la misma sección, ya que el efecto del pandeo haría que en cualquier caso el cordón superior tenga una sección mayor.

(4) QUINTAS, V.

"Diseño óptimo de pórticos formados por perfiles laminados rectos de sección constante" Informes de la Construcción. Vol. 39, n. 392.

(5) QUINTAS, V.

"Sobre el teorema de Maxwell y la optimización de arcos de cubierta" Informes de la Construcción. Vol. 40, n. 400. 